\section{Analysis and Synthesis of Nonlinear Systems With Uncertain Initial Conditions}

\author{
G. Chesi, Senior Member, IEEE, and \\ Y. S. Hung, Senior Member, IEEE
}

\begin{abstract}
This technical note considers the problem of computing extremal values of the trajectories over a given set of initial conditions as well as finding output controllers minimizing these extremal values under timedomain constraints for nonlinear systems. It is shown that upper bounds of the sought extremal values as well as candidates of the sought controllers can be computed by solving a one-parameter sequence of bilinear matrix inequality (BMI) optimizations obtained through the square matricial representation (SMR) of polynomials. Moreover, a necessary and sufficient condition is proposed to establish the tightness of the found upper bound in spite of the conservatism introduced by the nonconvexity of BMI optimizations and the chosen degree of the Lyapunov function and relaxing polynomials.
\end{abstract}

Index Terms-Bilinear matrix inequality (BMI), nonlinear system, square matricial representation (SMR), tightness, uncertain initial condition.

\section{INTRODUCTION}

Stability is not the only important issue in the analysis and synthesis of closed-loop systems, in fact, it is often mandatory also to deal with time-domain constraints on the system signals. For linear systems, the analysis problem can be solved by exploiting closed-form solutions for the trajectory, while the synthesis problem with time-domain constraints can be addressed via frequency-domain constraints on weighted transfer functions or via model predictive control (MPC). For nonlinear systems, the problem is more involved. First, there do not exist closed-form solutions for the trajectory except few special cases. Second, the synthesis must deal with the nonlinearities of the state updating law and measurable output, which affect convergence and efficiency of strategies such as nonlinear MPC [1], [2]; and, clearly, the problem is even more difficult if the initial condition is unknown and if the nonlinearities are nonpolynomial. In this respect, it should be noted that approaches such as state augmentation and approximation techniques for recasting the nonpolynomial system into a polynomial system can easily yield conservative results.

In this technical note, a new approach to deal with time-domain constraints in the analysis and synthesis of nonlinear systems is proposed. In particular, the proposed framework considers the computation of extremal values of the trajectories over a given set of initial conditions and the computation of output controllers minimizing these extremal values under time-domain constraints. The approach is first described for systems whose state updating law depends polynomially on the state and affinely on the input, and then it is extended to deal with nonpolynomial dependence on the state by using truncated Taylor expansions and taking into account the worst case remainders. It is shown that upper bounds of the sought extremal values as well as candidates of

Manuscript received June 29, 2007; revised December 7, 2007. Published August 27, 2008 (projected). Recommended by Associate Editor M. Q. Xiao.

G. Chesi is with the Department of Electrical and Electronic Engineering, University of Hong Kong, Hong Kong (e-mail: chesi@eee.hku.hk).

Y. S. Hung is with the Department of Electrical and Electronic Engineering, University of Hong Kong, Hong Kong (e-mail: yshung@eee.hku.hk).

Color versions of one or more of the figures in this paper are available online at http://ieeexplore.iee.org.

Digital Object Identifier 10.1109/TAC.2008.921045 the sought controllers can be computed by solving a one-parameter sequence of bilinear matrix inequality (BMI) optimizations by using Lyapunov functions (LFs) and polynomial relaxations based on the square matricial representation (SMR). In order to deal with the conservatism introduced by the nonconvexity of the problem as well as the chosen degree of the LF, a necessary and sufficient condition is proposed for the analysis part to establish the tightness of the found upper bound. A preliminary version of this technical note appeared in [12].

\section{PRELIMINARIES}

The notation is as follows. $\mathbb{N}, \mathbb{R}$ : natural and real numbers sets; $\mathcal{C}^{n}(\mathcal{X})$ : set of functions whose first $n$ derivatives are continuous over the set $\mathcal{X} ; \partial \mathcal{X}$ : boundary of set $\mathcal{X} ; 0_{n}$ : origin of $\mathbb{R}^{n} ; I$ : identity matrix (of size specified by the context); $\Phi^{\prime}$ : transpose of matrix $\Phi ; \Phi>0(\Phi \geq 0)$ : symmetric positive-definite (semidefinite) matrix $\Phi ; \phi>0(\phi \geq 0)$ : positive (nonnegative) entries vector $\phi ;\left.\phi\right|_{\max }: \max \left\{\phi_{1}, \ldots, \phi_{n}\right\}$ for $\phi \in \mathbb{R}^{n}$.

Consider the class of continuous-time systems

$$
\left\{\begin{array}{l}
\dot{x}(t)=f(x(t))+\varphi(x(t))+g(x(t)) u(t) \\
y(t)=h(x(t)) \\
x(0)=x_{\text {init }}
\end{array}\right.
$$

where $x(t) \in \mathbb{R}^{n}$ is the state, $u(t) \in \mathbb{R}^{n_{u}}$ is the input, $y(t) \in \mathbb{R}^{n} y$ is the output, $x_{\text {init }}$ is the initial condition, $f(x(t)), g(x(t))$, and $h(x(t))$ are polynomial functions of $x(t)$, and $\varphi(x(t))$ is a nonpolynomial function of $x(t)$. The set of initial conditions of interest is defined as

$$
\mathcal{A}=\left\{x \in \mathbb{R}^{n}: a_{i}(x) \geq 0 \forall i=1, \ldots, n_{a}\right\}
$$

where $a_{1}(x), \ldots, a_{n_{a}}(x)$ are polynomials. We assume that $\mathcal{A}$ is compact and define the set

$$
\begin{gathered}
\mathcal{K}=\left\{k(y): \mathbb{R}^{n_{y}} \rightarrow \mathbb{R}^{n_{u}} \text { such that, for } u(t)=k(y(t)),\right. \\
\lim _{t \rightarrow+\infty} x(t)=0_{n}, \text { and } w(t) \leq w_{0} \text { for all } \\
\left.t \in[0,+\infty) \text { and for all } x_{\text {init }} \in \mathcal{A}\right\}
\end{gathered}
$$

where $k$ is a polynomial function, $w(t) \in \mathbb{R}^{n} w$ is a selectable design signal to constrain the controller, and $w_{0} \in \mathbb{R}^{n} w$ is a given vector. Let us define the cost achieved by $u=k(y)$ as

$$
\sigma(k(y))=\left.\sup _{x_{\text {init }} \in \mathcal{A}, t \in[0,+\infty)} z(t)\right|_{\max }
$$

where $z(t) \in \mathbb{R}^{n} z$ is another selectable signal for optimization. The following is assumed:

1) the signals $z(t)$ and $w(t)$ are expressed by

$$
\begin{aligned}
z(t) & =\left[q_{z}(x(t))^{\prime}, l_{z}(u(t))^{\prime}\right]^{\prime} \\
w(t) & =\left[q_{w}(x(t))^{\prime}, l_{w}(u(t))^{\prime}\right]^{\prime}
\end{aligned}
$$

where $q_{z}, q_{w}$ are polynomial functions and $l_{z}, l_{w}$ are linear functions;

2) the origin is the equilibrium point of interest and the output vanishes in the origin.

The problems we address are as follows:

1) problem P1 (analysis): to compute the maximum of $\left.z(t)\right|_{\max }$ for the autonomous system

$$
\sigma=\sigma\left(0_{n_{u}}\right)
$$


2) problem P2 (synthesis): to compute the controller that minimizes the maximum of $\left.z(t)\right|_{\max }$

$$
\begin{aligned}
k^{*}(y) & =\arg \min _{k(y) \in \mathcal{K}} \sigma(k(y)) \\
\sigma^{*} & =\sigma\left(k^{*}(y)\right) .
\end{aligned}
$$

In the sequel, the dependence on the time $t$ will be generally omitted for ease of notation.

Let us observe that, depending on the choice of $z$, one can select several costs such as

$$
\begin{aligned}
z & =\left[h(x)^{\prime} B_{1}^{\prime} B_{1} h(x), u^{\prime} B_{2}^{\prime},-u^{\prime} B_{2}^{\prime}, B_{3} x\right]^{\prime} \\
\left.\Rightarrow z\right|_{\max } & =\max \left\{\left\|B_{1} y\right\|_{2}^{2},\left\|B_{2} u\right\|_{\infty}, b_{3,1} x, \ldots, b_{3, m} x\right\}
\end{aligned}
$$

where $B_{1}, B_{2}, B_{3}$ are weighting matrices of suitable dimensions, and $b_{3,1}, \ldots, b_{3, m}$ are the rows of $B_{5}$. Moreover, one can analogously define several constraints by similarly defining $w$ and $w_{0}$.

Before proceeding, let us introduce the complete SMR [3], [4]. Let $x^{\{m\}} \in \mathbb{R}^{\tau_{1}(m)}$ contain all monomials of degree not greater than $m$ in $x$, and let $p_{1}(x)$ be a polynomial of degree $2 \mathrm{~m}$. The complete SMR of $p_{1}(x)$ with respect to $x^{\{m\}}$ is

$$
p_{1}(x)=x^{\{m\}^{\prime}} P_{1}\left(\alpha_{1}\right) x^{\{m\}}
$$

with

$$
P_{1}\left(\alpha_{1}\right)=P_{1}+N_{1}\left(\alpha_{1}\right)
$$

where $P_{1}$ is any symmetric matrix such that $p_{1}(x)=$ $x^{\{m\}^{\prime}} P_{1} x^{\{m\}}, \alpha_{1} \in \mathbb{R}^{\nu_{1}(m)}$ is a vector of free parameters and $N_{1}\left(\alpha_{1}\right)$ is a linear parametrization of the set $\mathcal{N}_{1}(m)=\left\{N=N^{\prime}: x^{\{m\}^{\prime}} N x^{\{m\}}=0 \forall x\right\}$. As explained in [3] and [4] $\tau_{1}(m)=((n+m) !) /(n ! m !)$ and $\nu_{1}(m)=(1 / 2) \tau_{1}(m)\left(\tau_{1}(m)+1\right)-\tau_{1}(2 m)$.

In the case of polynomials having special structures, more compact representations can be derived. Indeed, polynomials without constant and linear terms can be represented with respect to the vector $x^{[m]} \in \mathbb{R}^{\tau_{2}(m)}$ containing all monomials of degree less than or equal to $m$ in $x$ but the constant term. For these polynomials, the SMR is analogously defined by substituting $\mathcal{N}_{1}(m)$ with the set $\mathcal{N}_{2}(m)=\{N=$ $\left.N^{\prime}: x^{[m]^{\prime}} m N x^{[m]}=0 \forall x\right\}$. We have $\tau_{2}(m)=\tau_{1}(m)-1$ and the dimension of $\mathcal{N}_{2}(m)$ is $\nu_{2}(m)=\nu_{1}(m)-\tau_{1}(m)+n+1$.

In the sequel, we will refer to $P_{1}$ and $P_{1}(\alpha)$ as SMR matrices of $p_{1}(x)$. Unless explicitly specified otherwise, it will be assumed that these matrices are defined with respect to $x^{\{m\}}$.

\section{ANALYSIS PROBLEM}

We consider first the case $\varphi(x)=0_{n}$. In order to simplify the description we assume the following.

A1) the linearized autonomous system is asymptotically stable, that is, $A$ is Hurwitz where

$$
A|B| C=\left.\frac{d(f(x)+\varphi(x))|d g(x)| d h(x)}{d x}\right|_{x=0_{n}} .
$$

The basic idea is to look for LFs whose unitary sublevel sets are invariant sets and contain $\mathcal{A}$. Then, we also require that these sublevel sets are contained in the region where the supremum of $\left.q_{z}(x)\right|_{\max }$ is bounded by a certain quantity $\zeta \in \mathbb{R}$. If we can find this LF, it is guaranteed that $\zeta \geq \sigma$. More formally, let us denote with $v: \mathbb{R}^{n} \rightarrow \mathbb{R}$ the LF candidate, and define with $\mathcal{V}(c)$ its sublevel set

$$
\begin{aligned}
\mathcal{V}(c) & =\left\{x \in \mathbb{R}^{n}: v(x) \leq c\right\} \\
\mathcal{Q}(\zeta) & =\left\{x \in \mathbb{R}^{n}:\left.q_{z}(x)\right|_{\max } \leq \zeta\right\} .
\end{aligned}
$$

Suppose there exists $v(x)$ and $\zeta \in \mathbb{R}$ such that the following holds.

C1) $v(x)$ is radially unbounded, $v(0)=0$ and $v(x)>0 \forall x \in$ $\mathbb{R}^{n} \backslash\left\{0_{n}\right\}$

C2) $\nabla v(x) f(x)<0 \forall x \in \mathcal{V}(1) \backslash\left\{0_{n}\right\}$;

C3) $\mathcal{A} \subseteq \mathcal{V}(1)$;

C4) $\mathcal{Q}(\zeta) \supseteq \mathcal{V}(1)$.

Then, $\zeta \geq \sigma$. Now, let us select $v(x)$ as a polynomial of degree $2 \delta_{v}$ and introduce

$$
\begin{aligned}
t_{1}(x) & =\nabla v(x) f(x)+s_{1}(x)(1-v(x)) \\
t_{2}(x) & =v(x)-1+\sum_{i=1}^{n_{a}} s_{2, i}(x) a_{i}(x) \\
t_{3, i}(x) & =s_{3, i}(x)\left(q_{z, i}(x)-\zeta\right)+1-v(x), \quad i=1, \ldots, n_{q_{z}}
\end{aligned}
$$

where $s_{*}(x)$ are auxiliary polynomials known as Hilbert's polynomials (see, for example, [5]-[7]).

Theorem 1: Let $\zeta>0$ be a given real scalar, and let $2 \delta_{v}$ be the chosen degree of $v(x)$. Let $V, S_{*}$, $T_{1}\left(V, S_{1}, \alpha_{1}\right), T_{2}\left(V, S_{2, *}, \alpha_{2}\right), T_{3, *}\left(V, S_{3, *}, \alpha_{3, *}\right) \quad$ be $\quad$ SMR matrices of the polynomials $v(x), s_{*}(x), t_{1}(x), t_{2}(x), t_{3, *}(x)$, respectively, with the matrices $V, S_{1}, T_{1}\left(V, S_{1}, \alpha_{1}\right)$ defined with respect to extended vectors without constant term. Define

$$
\begin{aligned}
& \lambda^{*}=\sup _{\lambda \in \mathbb{R}, V>0, S_{*}>0, \alpha_{*}} \lambda \text { s.t. }(15) \\
& \left\{\begin{array}{l}
T_{1}\left(V, S_{1}, \alpha_{1}\right)+\lambda I<0 \\
T_{2}\left(V, S_{2, *}, \alpha_{2}\right)+\lambda I \leq 0 \\
T_{3, i}\left(V, S_{3, i}, \alpha_{3, i}\right)+\lambda I \leq 0 \quad \forall i=1, \ldots, n_{q z} .
\end{array}\right.
\end{aligned}
$$

If $\lambda^{*} \geq 0$, then $\zeta \geq \sigma$.

Proof: Suppose that (15) is satisfied with $\lambda \geq 0$. Let $x^{\left\{\delta_{t, 1}\right\}}$ be the extended vector in the representation of $t_{1}(x)$. From the first inequality, we have

$$
0>x^{\left[\delta_{t, 1}\right]^{\prime}}\left(T_{1}\left(V, S_{1}, \alpha_{1}\right)+\lambda I\right) x^{\left[\delta_{t, 1}\right]}=t_{1}(x)+\lambda\left\|x^{\left[\delta_{t, 1}\right]}\right\|_{2}^{2}
$$

that is $t_{1}(x)<0$ for all $x \in \mathbb{R}^{n} \backslash\left\{0_{n}\right\}$. From the other inequalities, we similarly obtain that $v(x)>0$ and $s_{1}(x)>0$ for all $x \in \mathbb{R}^{n} \backslash\left\{0_{n}\right\}$, and that $t_{2}(x) \leq 0, t_{3, *}(x) \leq 0, s_{2, *}(x)>0$, and $s_{3, *}(x)>0$ for all $x$. Now, from $t_{1}(x)<0$ and $s_{1}(x)>0$, it follows that $\nabla v(x) f(x)<$ 0 for all $x \in \mathcal{V}(1) \backslash\left\{0_{n}\right\}$. Analogously, we prove that $x \in \mathcal{V}(1)$ for all $x \in \mathcal{A}$, and $x \in \mathcal{Q}(\zeta)$ for all $x \in \mathcal{V}(1)$. Moreover, $v(x)$ is radially unbounded because $V>0$, hence $\mathrm{C} 1-\mathrm{C} 4$ hold.

Theorem 1 provides a sufficient condition to establish if $\zeta$ is an upper bound of $\sigma$ by solving the optimization (14), which is a nonconvex optimization because the first inequality in (15) is a BMI because $T_{1}\left(V, S_{1}, \alpha_{1}\right)$ is a bilinear function of $V$ and $S_{1}$. BMI optimizations can be locally solved through dedicated software; alternatively, they can be approached via a sequence of convex LMI optimizations by alternatively fixing one variable and optimizing with respect to the other (we refer to this solution as $V-S$ iterations) as done in Section $\mathrm{V}$. In order to find $\sigma$, we can simply adopt a bisection algorithm minimizing $\zeta$ subject to the condition of Theorem I.

Now, we describe how Theorem 1 can be extended to deal with the case $\varphi(x) \neq 0_{n}$. First, it is worthwhile to observe that one may attempt to deal with this case by performing state augmentations to system into polynomial, or by substituting the nonpolynomial terms with their truncated Taylor expansions. However, it is known that these attempts can easily lead to conservative and disastrous results.

Our idea is to introduce truncated Taylor expansions taking into account the worst case remainder as proposed in [11] for estimating the 
domain of attraction. We suppose that assumptions A1 previously introduced and $\mathrm{A} 2$ below hold.

A2) the function $\varphi(x)$ has the form

$$
\varphi(x)=\sum_{i=1}^{r} p_{i}(x) \psi_{i}\left(x_{k_{i}}\right)
$$

where $p_{i}(x)$ are polynomials, $k_{1}, \ldots, k_{r}$ are integers in $[1, n]$, and $\psi_{i}: \mathcal{C}^{d_{i}}\left(\left[c_{i, \min }, c_{i, \max }\right]\right) \rightarrow \mathbb{R}$ are nonpolynomial functions for integers $d_{i} \geq 1$ and scalars $c_{i, \min }<0, c_{i, \max }>0$.

Let us write $\psi_{i}\left(x_{k_{i}}\right)$ via the Taylor expansion centered in $x_{k_{i}}=0$ up to the $\beta_{i}$ th power for a given integer $\beta_{i}$ in $\left[1, d_{i}-1\right]$ and express the remainder in the Lagrange form

$$
\psi_{i}\left(x_{k_{i}}\right)=\mu_{i}\left(x_{k_{i}}\right)+\varrho_{i}\left(x_{k_{i}}\right) \theta_{i}\left(b_{i}\right)
$$

where $\mu_{i}\left(x_{k_{i}}\right)$ is a polynomial of degree $\beta_{i}, \varrho_{i}\left(x_{k_{i}}\right)$ is a monomial of degree $\beta_{i}+1, \theta_{i}\left(b_{i}\right)$ is the $\beta_{i}+1$ th derivative of $\psi_{i}\left(x_{k_{i}}\right)$ evaluated for $x_{k_{i}}=b_{i}$, and $b_{i} \in\left[0, x_{k_{i}}\right]$ if $x_{k_{i}} \geq 0$ or $b_{i} \in\left[x_{k_{i}}, 0\right]$, otherwise. Let us introduce the polynomials

$$
\begin{aligned}
& t_{1, m}(x)= \nabla v(x)\left(f(x)+\sum_{i=1}^{r} p_{i}(x) \mu_{i}\left(x_{k_{i}}\right)\right) \\
&+s_{1, m}(x)(1-v(x))+\sum_{i=1}^{r}\left(\theta_{i, m_{i}}\right. \\
&\left.+(-1)^{m_{i}} s_{1, m, i}(x)\right) \nabla v(x) p_{i}(x) \varrho_{i}\left(x_{k_{i}}\right), \quad m \in\{0,1\}^{r} \\
& t_{4, i}(x)= s_{4, i}(x)\left(x_{k_{i}}-c_{i, \max }\right)\left(x_{k_{i}}-c_{i, \min }\right)+1-v(x), \\
& \quad i=1, \ldots, r \quad(18)
\end{aligned}
$$

where $s_{1, m}(x), s_{1, m, i}(x), s_{4, i}(x)$ are polynomials, and

$$
\theta_{i, m_{i}}=\left\{\begin{array}{ll}
\sup _{b_{i} \in\left[c_{i, \min }, c_{i, \max }\right]} \theta_{i}\left(b_{i}\right), & \text { if } m_{i}=0 \\
\inf _{b_{i} \in\left[c_{i, \min }, c_{i, \max }\right]} \theta_{i}\left(b_{i}\right), & \text { if } m_{i}=1
\end{array} .\right.
$$

Theorem 2: Let $\zeta>0$ be a given real scalar, and let $2 \delta_{v}$ be the chosen degree of $v(x)$. Let $S_{*}, T_{1, m}\left(V, S_{1, *}, \alpha_{1, m}\right), T_{4, i}\left(V, S_{4, i}, \alpha_{4, i}\right)$ be SMR matrices of the polynomials $s_{*}(x), t_{1, m}(x), t_{4, i}(x)$, respectively. Define

$$
\begin{aligned}
& \lambda^{*}=\sup _{\lambda \in \mathbb{R}, V>0, S_{*}>0, \alpha_{*}} \lambda \text { s.t. (21) } \\
& \left\{\begin{array}{l}
\text { inequality }(15) \text { without the first inequality } \\
T_{1, m}\left(V, S_{1, *}, \alpha_{1, m}\right)+\lambda I<0 \quad \forall m \in\{0,1\}^{r} \\
T_{4, i}\left(V, S_{4, i}, \alpha_{4, i}\right)+\lambda I \leq 0 \quad \forall i=1, \ldots, r .
\end{array}\right.
\end{aligned}
$$

If $\lambda^{*} \geq 0$, then $\zeta \geq \sigma$.

Proof: Suppose that (21) is satisfied with $\lambda \geq 0$. Let $\bar{x}$ be any point in $\mathcal{V}(1)$ and define

$$
\bar{m}=\left[\bar{m}_{1}, \ldots, \bar{m}_{r}\right]^{\prime}, \bar{m}_{i}= \begin{cases}0, & \text { if } \nabla v(\bar{x}) p_{i}(\bar{x}) \varrho_{i}\left(\bar{x}_{k_{i}}\right) \geq 0 \\ 1, & \text { otherwise }\end{cases}
$$

From $t_{4, i}(\bar{x}) \leq 0$ it follows that $\bar{x}_{k_{i}} \in\left[c_{i, \max }, c_{i, \max }\right]$. Moreover, because $s_{1, \bar{m}}(\bar{x})>0$ and $s_{1, \bar{m}, i}(\bar{x})>0$, from $t_{1, \bar{m}}(\bar{x})<0$, we have

$$
\begin{aligned}
0> & \nabla v(\bar{x})\left(f(\bar{x})+\sum_{i=1}^{r} p_{i}(\bar{x}) \mu_{i}\left(\bar{x}_{k_{i}}\right)\right)+s_{1, \bar{m}}(\bar{x})(1-v(\bar{x})) \\
& +\sum_{i=1}^{r}\left(\theta_{i, \bar{m}_{i}}+(-1)^{\bar{m}_{i}} s_{1, \bar{m}, i}(x)\right) \nabla v(\bar{x}) p_{i}(\bar{x}) \varrho_{i}\left(\bar{x}_{k_{i}}\right)
\end{aligned}
$$

$$
\begin{aligned}
> & \nabla v(\bar{x})\left(f(\bar{x})+\sum_{i=1}^{r} p_{i}(\bar{x}) \mu_{i}\left(\bar{x}_{k_{i}}\right)\right) \\
& +\sum_{i=1}^{r} \theta_{i, \bar{m}_{i}} \nabla v(\bar{x}) p_{i}(\bar{x}) \varrho_{i}\left(\bar{x}_{k_{i}}\right) \\
= & \nabla v(\bar{x})(f(\bar{x})+\varphi(\bar{x}))
\end{aligned}
$$

that is $\dot{v}(\bar{x})<0$. Following the remaining proof of Theorem 1, we conclude that $\zeta \geq \sigma$.

Theorem 2 provides a sufficient condition for computing an upper bound of $\sigma$ through BMI optimizations in spite of the presence of nonpolynomial terms in (1). This is made possible by taking into account the worst case remainder of the truncated Taylor expansion in the time-derivative of $v(x)$. In particular, the unitary sublevel set $\mathcal{V}(1)$ is constrained within the set

$$
\mathcal{C}=\left\{x \in \mathbb{R}^{n}: x_{k_{i}} \in\left[c_{i, \min }, c_{i, \max }\right], i=1, \ldots, r\right\}
$$

where $c_{i, \min }, c_{i, \max }$ delimit the region of validity of the worst case remainder computed through the bounds $\theta_{i, m_{i}}$. If no information are available a priori about the extension of $\mathcal{V}(1)$ and/or the computation of $\theta_{i, m_{i}}$ is difficult, conservative estimates of $c_{i, \min }, c_{i, \max }, \theta_{i, m_{i}}$ can be used. In fact, let us observe that the effect of these conservative quantities, represented by term

$$
\omega_{i, m_{i}}(x)=\theta_{i, m_{i}} \nabla v(x) p_{i}(x) \varrho_{i}\left(x_{k_{i}}\right)
$$

in the polynomials $t_{1, m}(x)$, can be compensated by increasing the degree $\beta_{i}$ of the truncated Taylor expansion, which makes this term convergent to zero.

\section{Synthesis AND TightNess PROBlEMS}

Let us consider first problem P2. In order to simplify the description, we assume the following:

A3) the linearized system can be asymptotically stabilized through a static output feedback, i.e., there exists $K \in \mathbb{R}^{n_{i} \times n_{u}}$ such that

$A+B K C$ is Hurwitz where $A, B, C$ are as in (10).

Let us express the controller $k(y)$ in the class $\mathcal{K}$ in (3) as

$$
k(y)=\xi^{\prime} y^{\left[\delta_{k}\right]}
$$

where $\delta_{k}$ is the degree of the controller, and $\xi \in \mathbb{R}^{\tau_{2}\left(\delta_{k}\right)}$ is the coefficient vector to be determined. Because the state updating law depends linearly on the input, one can exploit the condition derived in Theorem 2 by letting the vector $\xi$ vary together with the other variables of the optimization (20). In particular, this can be done by adding $t_{1, u}(x)$ to $t_{1, m}(x)$, where

$$
t_{1, u}(x)=\nabla v(x) g(x) \xi^{\prime} y^{\left[\delta_{k}\right]} .
$$

Then, in order to take into account the presence of the input in the cost signal $z$, we introduce for $i=1, \ldots, n_{l z}$

$$
t_{5, i}(x)=l_{z, i}\left(\xi^{\prime}(h(x))^{\left[\delta_{k}\right]}\right)-\zeta+s_{4, i}(x)(1-v(x)) .
$$

In order to take into account the time-domain constraint on the signal $w$, we also define the polynomials $t_{j, *}$ and $s_{j, *}$ for $j=6,7$ analogous to those for $j=3,5$ for the cost signal $z$ by replacing $q_{z, i}, l_{z, i}, \zeta$ with $q_{w, i}, l_{w, i}, w_{0, i}$, respectively.

Theorem 3: Let $\zeta>0$ be a given real scalar, and let $2 \delta_{v}$ be the chosen degree of $v(x)$. Let $T_{1, u}(V, \xi), S_{*}, T_{5, *}\left(V, \xi, S_{5, *}, \alpha_{5, *}\right), T_{6, *}\left(V, S_{6, *}, \alpha_{6, *}\right), T_{7, *}$ $\left(V, \xi, S_{7, *}, \alpha_{7, *}\right)$ be SMR matrices of the polynomials $t_{1, u}(x), s_{*}, t_{5, *}(x), t_{6, *}(x), t_{7, *}(x)$, respectively, with $T_{1, u}(V, \xi)$ 
defined with respect to the extended vector used for defining $T_{1}\left(V, S_{1}, \alpha_{1}\right)$. Define

$$
\begin{gathered}
\lambda^{*}=\sup _{\lambda \in \mathbb{R}, V>0, \xi, S_{*}>0, \alpha *} \lambda \text { s.t. }(28) \\
\left\{\begin{array}{l}
\text { ineq. }(21) \text { appending } T_{1, u}(V, \xi) \text { to } T_{1, m}\left(V, S_{1, *}, \alpha_{1, m}\right) \\
T_{5, i}\left(V, \xi, S_{5, i}, \alpha_{5, i}\right)+\lambda I \leq 0 \quad \forall i=1, \ldots, n_{l_{z}} \\
T_{6, i}\left(V, S_{6, i}, \alpha_{6, i}\right)+\lambda I \leq 0 \quad \forall i=1, \ldots, n_{q_{w}} \\
T_{7, i}\left(V, \xi, S_{7, i}, \alpha_{7, i}\right)+\lambda I \leq 0 \quad \forall i=1, \ldots, n_{l_{w}} .
\end{array}\right.
\end{gathered}
$$

If $\lambda^{*} \geq 0$, then $\zeta \geq \sigma^{*}$.

Proof: It is analogous to the proof of Theorem 2 by observing that (28) implies $t_{1, m}(x)+t_{1, u}(x)<0$. Hence, we have $\nabla v(x)(f(x)+$ $g(x) \xi^{\prime}(h(x))^{\left[\delta_{k}\right]}<0$ for all $x \in \mathcal{V}(1) \backslash\left\{0_{n}\right\}$. Moreover, from (28), we have $l_{z} \mid \max \left(\xi^{\prime}(h(x))^{\left.\left[\delta_{k}\right)\right]} \leq \zeta\right.$ and $\left[q_{w}(x)^{\prime}, l_{w}\left(\xi^{\prime}(h(x))^{\left[\delta_{k}\right]}\right)^{\prime}\right]^{\prime} \leq w_{0}$ for all $x \in \mathcal{V}(1)$. Therefore, $\mathcal{V}(1)$ is an invariant set for the controlled system where $\left.z\right|_{\max } \leq \zeta$ and $w \leq w_{0}$.

Theorem 3 allows one to compute an upper bound of $\sigma^{*}$ and a controller guaranteeing this upper bound by solving the optimization (27). In the sequel, we will indicate with $\zeta_{2 \delta_{v}, \delta_{k}}$ the best upper bound of $\sigma^{*}$ found with an LF of degree $2 \delta_{v}$ and a controller of degree $\delta_{k}$.

Now, we consider the problem of establishing if the best upper bound found for $\sigma$ by using Theorem 2 with an LF of degree $2 \delta_{v}$ is tight or not. Let us indicate with $\zeta_{2} \delta_{v}$ this upper bound, and let us observe that $\zeta_{2 \delta_{v}}$ can be conservative for the following several reasons:

- the degree of the LF or auxiliary polynomials $s_{*}(x)$ is too low;

- the degree of the Taylor expansion for $\varphi(x)$ is too low;

- BMI optimizations are nonconvex.

The following result provides a necessary and sufficient condition to establish if $\zeta_{2} \delta_{v}$ is tight.

Theorem 4: Let $V_{2 \delta_{v}}$ be the optimal value of $V$ corresponding to the found $\zeta_{2 \delta_{v}}$, and let $v_{2} \delta_{v}(x)$ and $\mathcal{V}_{2 \delta_{v}}(1)$ be the LF and its unitary sublevel set corresponding to $V_{2} \delta_{v}$. Then

$$
\zeta_{2 \delta_{v}}=\sigma \Longleftrightarrow \exists x_{R, \text { init }} \in \mathcal{T}, \bar{t} \in[0, \infty): x_{R}(\bar{t}) \in \mathcal{A}
$$

where

$$
\mathcal{T}=\partial \mathcal{V}_{2 \delta_{v}}(1) \cap \partial \mathcal{Q}\left(\zeta_{2 \delta_{v}}\right)
$$

and $x_{R}(t)$ is the solution of the system

$$
\left\{\begin{array}{l}
\dot{x}_{R}(t)=-f\left(x_{R}(t)\right)-\varphi\left(x_{R}(t)\right) \\
x_{R}(0)=x_{R, \text { init }} .
\end{array}\right.
$$

Proof: " $\Leftarrow$ " Suppose that $\exists x_{R}$,init $\in \mathcal{T}$ and $\bar{t} \in[0, \infty)$ such that $x_{R}(\bar{t}) \in \mathcal{A}$. Then, because the system (31) evolves reversely with respect to the system (1), it follows that by initializing (1) with $x_{\text {init }}=x_{R}(\bar{t})$, we have that $x(\bar{t})=x_{R \text {,init. }}$. Because $x_{\text {init }} \in \mathcal{A}$, it follows from the definition of $\sigma$ in (6) that $\left.z(\bar{t})\right|_{\max } \leq \sigma$. On the other hand, $\left.z(\bar{t})\right|_{\max }=\left.q_{z}(x(\bar{t}))\right|_{\max }=\left.q_{z}\left(x_{R, \text { init }}\right)\right|_{\max }=\zeta_{2} \delta_{v}$ because $x_{R \text {,init }} \in \partial \mathcal{Q}\left(\zeta_{2 \delta_{v}}\right)$, and hence, $\zeta_{2 \delta_{v}} \leq \sigma$. However, $\zeta_{2 \delta_{v}} \geq \sigma$. Therefore, $\zeta_{2} \delta_{v}=\sigma$.

" $\Rightarrow$ " Suppose that $\zeta_{2 \delta_{v}}=\sigma$. From the definition of $\sigma$ in (6) and because $\mathcal{A}$ is compact, it follows that $\exists x_{\text {init }} \in \mathcal{A}$ and $\bar{t} \in[0, \infty)$ such that $\left.z(\bar{t})\right|_{\max }=\sigma$. Observe that $x(\bar{t}) \in \partial \mathcal{Q}\left(\zeta_{2 \delta_{v}}\right)$ because $\zeta_{2 \delta_{v}}=\sigma$. Suppose now for contradiction that $x(\bar{t})$ does not belong to $\partial \mathcal{V}_{2 \delta_{v}}(1)$. Then, this implies either that $v_{2 \delta_{v}}(x(\bar{t}))>1$ or that $v_{2 \delta_{v}}(x(\bar{t}))<1$. However, the former implies that $x(\bar{t})$ lies outside an invariant set containing the initial condition $x_{\text {init }} \in \mathcal{A}$, and the second implies that $\mathcal{V}_{2 \delta_{v}}(1)$ is not included in $\mathcal{Q}\left(\zeta_{2 \delta_{v}}\right)$ as ensured by the third inequality in (15). Hence, both hypotheses are impossible and hence $x(\bar{t}) \in \partial \mathcal{V}_{2 \delta_{v}}(1)$. Therefore, $x(\bar{t}) \in \mathcal{T}$. Then, by initializing the reverse system (31) with $x_{R \text {,init }}=x(\bar{t})$, we obtain that $x_{R}(\bar{t})=x_{\text {init }} \in \mathcal{A}$, which concludes the proof

The condition of Theorem 4 can be checked in two steps: 1) computing $\mathcal{T}$, which is the intersection of the boundaries of $\mathcal{V}_{2 \delta_{v}}(1)$ and $\mathcal{Q}\left(\zeta_{2 \delta_{v}}\right)$; and 2) computing the trajectories of the reverse system (31) initialized with the points in $\mathcal{T}$ and checking if at least one of these trajectories intersects $\mathcal{A}$. Observe that $\mathcal{T}$ is composed by a finite number of points, typically one, being the intersection of two tangent surfaces. The set $\mathcal{T}$ can be found by solving

$$
\left\{\begin{array}{l}
v_{2} \delta_{v}(x)-1=0 \\
\left.q_{z}(x)\right|_{\max }-\zeta_{2 \delta_{v}}=0 .
\end{array}\right.
$$

However, solving the system (32) can be a difficult task because it is a nonlinear system.

Theorem 5: Define

$$
M_{i}=T_{3, i}\left(V_{2 \delta_{v}}, S_{3, i, 2 \delta_{v}}, \alpha_{3, i, 2 \delta_{v}}\right), \quad i=1, \ldots, n_{q_{z}}
$$

where $S_{3, i, 2 \delta_{v}}$ and $\alpha_{3, i, 2 \delta_{v}}$ are the found optimal values of $S_{3, i}$ and $\alpha_{3, i}$. Then

$$
\begin{aligned}
\mathcal{T} & =\left\{x \in \bigcup_{i=1}^{n_{q z}} \mathcal{M}_{i}: x \text { satisfies }(32)\right\} \\
\mathcal{M}_{i} & =\left\{x \in \mathbb{R}^{n}: x^{\left\{\delta_{t, 3}\right\}} \in \operatorname{ker}\left(M_{i}\right)\right\} .
\end{aligned}
$$

Proof: Consider $x \in \mathcal{T}$. Because $x$ satisfies (32), there exists $j$ such that $t_{3, j}(x)=0$. Now, from (21), it follows that $M_{j} \leq 0$. Hence $0=t_{3, j}(x)=x^{\left\{\delta_{t, 3}\right\}^{\prime}} M_{j} x^{\left\{\delta_{t, 3}\right\}}=x^{\left\{\delta_{t, 3}\right\}^{\prime}}\left(-\tilde{M}_{j}^{\prime} \tilde{M}_{j}\right) x^{\left\{\delta_{t, 3}\right\}}=$ $-\left\|\tilde{M}_{j} x^{\left\{\delta_{t, 3}\right\}}\right\|_{2}^{2}$, where $\tilde{M}_{j}$ is any Cholesky factor of $-M_{j}$. Clearly, $\left\|\tilde{M}_{j} x^{\left\{\delta_{t, 3}\right\}}\right\|_{2}^{2}=0$ if and only if $x^{\left\{\delta_{t, 3}\right\}} \in \operatorname{ker}\left(\tilde{M}_{j}\right)$. Because $\operatorname{ker}\left(\tilde{M}_{j}\right)=\operatorname{ker}\left(M_{j}\right)$, we conclude the proof.

Theorem 5 provides an alternative way of computing the set $\mathcal{T}$, which consists of finding the vectors $x \in \mathbb{R}^{n}$ satisfying $x^{\left\{\delta_{t, 3}\right\}} \in$ $\operatorname{ker}\left(M_{i}\right)$ for $i=1, \ldots, n_{q_{z}}$. This can be trivially done if the dimension of $\operatorname{ker}\left(M_{i}\right)$ is 1 corresponding to a unique element in the set $\mathcal{M}_{i}$ as shown in Example 1 in Section V. In other cases, one can use, for example, the approaches described in [8]-[10]. Once the sets $\mathcal{M}_{i}$ have been found, one can simply obtain the set $\mathcal{T}$ by verifying if the vectors contained in $\bigcup_{i=1}^{n} q_{z} \mathcal{M}_{i}$ satisfy the equations in (32) via trivial substitution.

Before introducing some examples in the next section, let us observe that the BMI optimization can be initialized with any matrix $K$ satisfying assumption A3) and any LF proving the asymptotical stability of the linearized system controlled by such a matrix.

\section{EXAMPLES}

\section{Example 1}

Let us consider the nonpolynomial system

$$
\left\{\begin{array}{l}
\dot{x}_{1}=x_{2}+0.2 x_{1}^{2} x_{2}+0.2\left(1-e^{x_{1}}\right) \\
\dot{x}_{2}=-1.5 x_{1}-2 x_{2}-1.1 x_{2}^{2}+u \\
y=x_{1} \\
x_{\text {init }} \in \mathcal{A}=\left\{x:\|x\|_{2}^{2} \leq 4\right\}
\end{array}\right.
$$

whose trajectories are shown in Fig. 1(a). We consider the following synthesis problem:

$$
\arg \min _{\text {stabilizing } u(t)=k(y(t)),|u(t)|<3} \sup _{x_{\text {init }} \in \mathcal{A}, t \in[0,+\infty)}\|x(t)\|_{\infty} .
$$




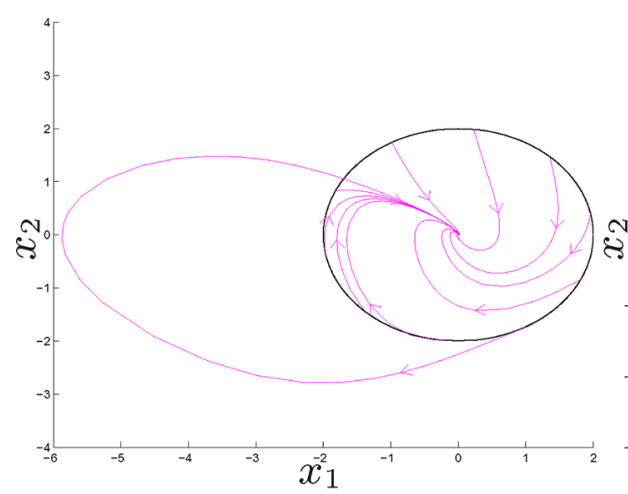

(a)

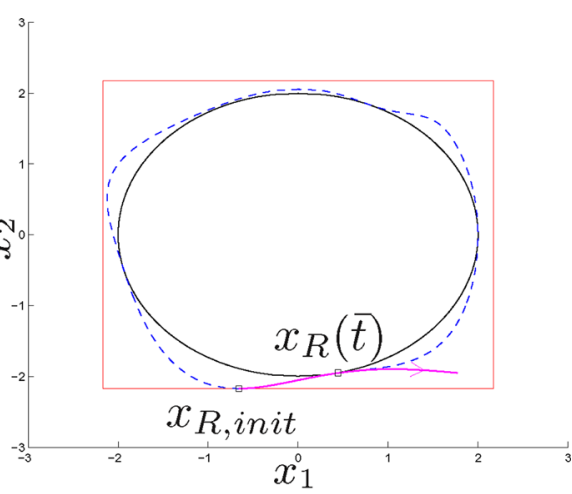

(b)

Fig. 1. Example 1. (a) Uncontrolled system trajectories starting in the set of initial conditions $\mathcal{A}$. (b) System controlled with $u=k_{6,3}(y)$ : the trajectories are confined in the unitary sublevel set $\mathcal{V}(1)$ (dashed curve) included in the region $\left\{x:\|x\|_{\infty}<\zeta_{6,3}\right\}$ (box). The trajectory $x_{R}(t)$ intersects $\mathcal{A}$, which means that $\zeta_{6,3}$ is tight.

TABLE I

EXAMPLE 1: UPPER BOUNDS $\zeta_{2 \delta_{v}, \delta_{k}}$ COMPUTED THROUGH SIMPLE $V$-S Iterations

\begin{tabular}{c|c|c|c}
$2 \delta_{v} \backslash \delta_{k}$ & 1 & 2 & 3 \\
\hline 2 & $+\infty$ & $+\infty$ & $+\infty$ \\
\hline 4 & $+\infty$ & 2.727 & 2.687 \\
\hline 6 & $+\infty$ & 2.463 & 2.191
\end{tabular}

This problem can be reformulated as in (7) with

$$
\begin{aligned}
f(x) & =\left[\begin{array}{c}
0.2+x_{2}+0.2 x_{1}^{2} x_{2} \\
-1.5 x_{1}-2 x_{2}-1.1 x_{2}^{2}
\end{array}\right] \quad \varphi(x)=\left[\begin{array}{c}
-0.2 e^{x_{1}} \\
0
\end{array}\right] \\
g(x) & =\left[\begin{array}{l}
0 \\
1
\end{array}\right] \quad h(x)=[1,0] \quad a_{1}=4-x_{1}^{2}-x_{2}^{2} \\
z & =\left[x^{\prime},-x^{\prime}\right]^{\prime} \quad w=[u,-u]^{\prime} \quad w_{0}=[3,3]^{\prime} .
\end{aligned}
$$

Let us use Theorem 3. We express $\varphi(x)$ as in (16) and (17) with $\beta_{1}=4$ obtaining

$$
\begin{aligned}
\varphi(x) & =p_{1}(x) \psi_{1}\left(x_{1}\right) \quad p_{1}(x)=[-0.2,0]^{\prime} \\
\psi_{1}\left(x_{1}\right) & =e^{x_{1}} \\
\mu_{1}\left(x_{1}\right) & =\sum_{i=0}^{4} \frac{x_{1}^{i}}{i !} \quad \varrho_{1}\left(x_{1}\right)=\frac{x_{1}^{5}}{5 !} \quad \theta_{1}\left(b_{1}\right)=e^{b_{1}} .
\end{aligned}
$$

The bounds for the remainder $\theta_{1}\left(b_{1}\right)$ are derived as in (19) with the choice $c_{1, \min }=-\zeta$ and $c_{1, \max }=\zeta$ (where $\zeta$ is the candidate upper bound) obtaining $\theta_{1,0}=e^{\zeta}$ and $\theta_{1,1}=e^{-\zeta}$. Table I shows the upper bounds $\zeta_{2} \delta_{v}, \delta_{k}$ computed through simple $V-S$ iterations. The controller corresponding to $\zeta_{6,3}$ is $k_{6,3}(y)=0.900 y+0.918 y^{2}-0.184 y^{3}$.

Let us consider the problem of establishing if the upper bound $\zeta_{6,3}$ is tight for the system controlled with $u=k_{6,3}(y)$. This can be done by using Theorems 4 and 5 . We have that $\operatorname{ker}\left(M_{1}\right)=\emptyset$ and $\operatorname{ker}\left(M_{2}\right)$ is a linear space of dimension one. There exists only $\bar{x}=[-0.665,-2.191]^{\prime}$ such that $\bar{x}^{\{3\}}=d$ and hence $\mathcal{M}_{2}=\{\bar{x}\}$. From (34), one obtains that $\mathcal{T}=\{\bar{x}\}$. The trajectory $x_{R}(t)$ of (31) with initial condition $x_{R \text {,init }}=\bar{x}$ intersects $\mathcal{A}$ in $x_{R}(\bar{t})$ as shown in Fig. 1(b). Therefore, the upper bound is tight.

\section{Example 2}

Consider the inverted pendulum controlled with a DC motor in Fig. 2(a)

$$
\left\{\begin{array}{l}
\dot{x}_{1}=x_{2} \\
\dot{x}_{2}=\left(I+m L^{2}\right)^{-1}\left(-b x_{2}+K_{t} x_{3}+m g L \sin x_{1}\right) \\
\dot{x}_{3}=L_{a}^{-1}\left(-K_{e} x_{2}-R_{a} x_{3}+u\right) \\
y=x_{1}
\end{array}\right.
$$

where $x_{1}$ is the angle, $x_{2}$ is the angular speed, $x_{3}$ is the armature current, $u$ is the input voltage, $b=0.5 \mathrm{~J} \cdot \mathrm{s} \cdot \mathrm{rad}^{-1}$ is the viscous friction coefficient, $I=0.03 \mathrm{~kg} \cdot \mathrm{m}^{2}$ is the moment of inertia of the pole, $m=$ $0.1 \mathrm{~kg}$ is the mass of the pole, $L=0.5 \mathrm{~m}$ is the distance from the center of gravity to the pivot, $K_{t}=1 \mathrm{~J} \cdot \mathrm{A}^{-1}$ is the torque constant, $K_{e}=0.01 \mathrm{v} \cdot \mathrm{s} \cdot \mathrm{rad}^{-1}$ is the electric constant, $L_{a}=0.001 \mathrm{H}$ is the inductance, $R_{a}=0.1 \mathrm{Ohm}$ is the resistance, and $g$ is the gravitational constant. We consider the synthesis problem

$$
\begin{aligned}
& \arg \underset{\text { stabilizing } u(t)=k(y(t))}{\min _{x_{\text {init }} \in \mathcal{A}, t \in[0,+\infty)}|u(t)|} \\
& \mathcal{A}=\left\{x:\left(\frac{x_{1}}{\pi / 2}\right)^{2}+\left(\frac{x_{2}}{0.1}\right)^{2}+\left(\frac{x_{3}}{0.1}\right)^{2} \leq 1\right\} .
\end{aligned}
$$

Let us express $\psi_{1}\left(x_{1}\right)=\sin x_{1}$ as in (17) with $\beta_{1}=4$ obtaining

$$
\mu_{1}\left(x_{1}\right)=x_{1}-\frac{x_{1}^{3}}{3 !} \quad \varrho_{1}\left(x_{1}\right)=\frac{x_{1}^{5}}{5 !} \quad \theta_{1}\left(b_{1}\right)=\cos b_{1} .
$$

The bounds in (19) for the remainder $\theta_{1}\left(b_{1}\right)$ are simply selected as $\theta_{1,0}=1$ and $\theta_{1,1}=-1$. In order to initialize our procedure, we observe that the linearized system can be stabilized by $u=k_{\operatorname{lin}} y$ with $k_{\text {lin }} \in(-6.54,-0.049)$ and select, for example, $K=-1$. Table II(a) shows the upper bounds $\zeta_{2} \delta_{v}, \delta_{k}$ computed again through $V-S$ iterations. The controller corresponding to $\zeta_{4,3}$ is $k_{4,3}(y)=-0.0490 y+$ $0.0071 y^{3}$ whose performance is illustrated in Fig. 2(b) and (c).

Let us observe from Fig. 2(c) that the convergence can be quite slow. This happens because the linearized controlled system is almost marginally stable only. In order to cope with this problem, we can repeat the procedure by imposing that the maximum real part of the eigenvalues of the linearized controlled system is less than a negative value, for example, -0.4 . From standard root locus investigation, this is equivalent to adding the LMIs $\xi_{1}>-5.977$ and $\xi_{1}<-0.072$ in the optimization, where $\xi_{1}$ is the coefficient of the linear term of the controller. Table II(b) shows the obtained upper bounds, and Fig. 2(d) shows the corresponding performance. Observe that the convergence 


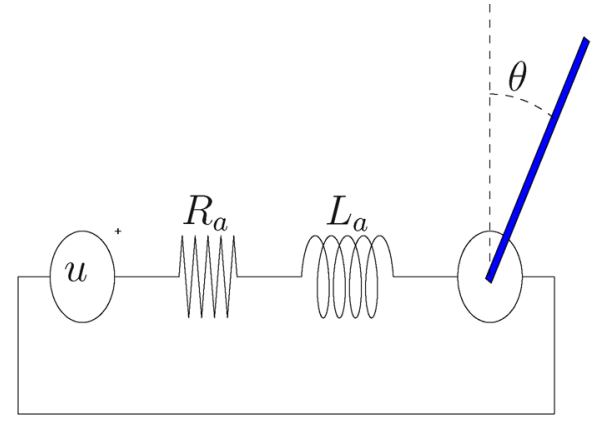

(a)

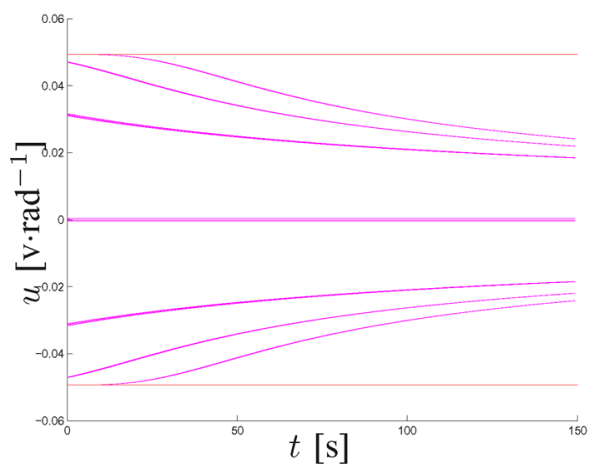

(c)

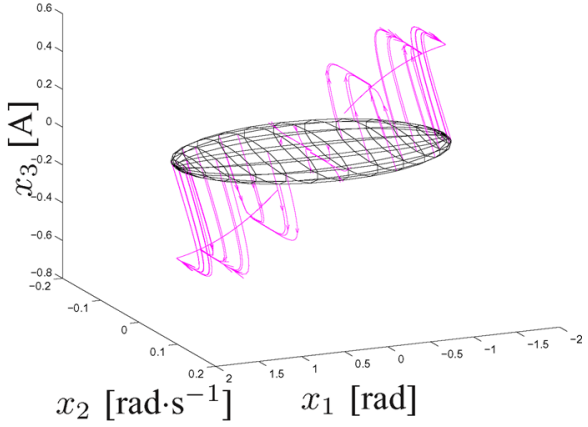

(b)

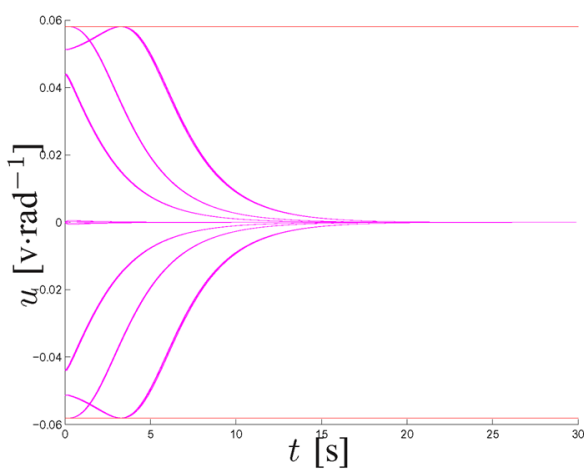

(d)

Fig. 2. Example 2. (a) Inverted pendulum controlled with a DC motor. (b) Trajectories starting in $\mathcal{A}$ with $u=k_{4,3}(y)$. (c) Control input with $u=k_{4,3}(y)$. (d) Control input achieved by constraining the linear term of the controller.

TABLE II

EXAMPLE 2: UPPER BOUNDS $\zeta_{2 \delta_{v}, \delta_{k}}$ OBTAINED WITH (A) UNCONSTRAINED AND (B) CONSTRAINED CONTROLLER LINEAR TERM

\begin{tabular}{c|c|c|c}
$2 \delta_{v} \backslash \delta_{k}$ & 1 & 2 & 3 \\
\hline 2 & 0.0783 & 0.0783 & 0.0503 \\
\hline 4 & 0.0772 & 0.0772 & 0.0494
\end{tabular}

is much faster, clearly at the expense of a larger amplitude of the control signal.

\section{CONCLUSION}

The problem of computing extremal values of the trajectories over a given set of initial conditions and the problem of computing output controllers minimizing these extremal values under time-domain constraints have been addressed for polynomial and nonpolynomial systems. It has been shown that an upper bound of the sought extremal values as well as candidates of the sought controllers can be found by solving a one-parameter sequence of BMI optimizations, which can be approached through either recently developed software or simple iterative convex LMI optimizations. Because the found upper bound may be conservative due to the nonconvexity of BMI optimizations and the chosen degree of the Lyapunov function, a necessary and sufficient condition has been proposed for the analysis part to establish the tightness of this upper bound in spite of all these sources of conservatism.

\section{ACKNOWLEDGMENT}

The authors would like to thank the Associate Editor and the reviewers for their time and comments.

\section{REFERENCES}

[1] F. Allgower and A. Zheng, Nonlinear Model Predictive Control: Assessment and Future Directions for Research, ser. Progress in Systems and Control Series. Basel, Switzerland: Birkhauser Verlag, 2000.

[2] B. Kouvaritakis and M. Cannon, Non-Linear Predictive Control: Theory and Practice, ser. IEE Control Series. London, U.K.: Institute of Electrical Engineers, 2001

[3] G. Chesi, A. Tesi, A. Vicino, and R. Genesio, "On convexification of some minimum distance problems," in Proc. 5th Eur. Control Conf., Karlsruhe, Germany, 1999.

[4] G. Chesi, A. Garulli, A. Tesi, and A. Vicino, "Solving quadratic distance problems: An LMI-based approach," IEEE Trans. Autom. Control, vol. 48, no. 2, pp. 200-212, Feb. 2003.

[5] J. Bochnak, M. Coste, and M.-F. Roy, Real Algebraic Geometry. New York: Springer-Verlag, 1998.

[6] M. Putinar, "Positive polynomials on compact semi-algebraic sets," Ind. Univ. Math., vol. 42, pp. 969-984, 1993.

[7] G. Stengle, "A Nullstellensatz and a Positivstellensatz in semialgebraic geometry," Math. Ann., vol. 207, pp. 87-97, 1974.

[8] G. Chesi, A. Garulli, A. Tesi, and A. Vicino, "An LMI-based approach for characterizing the solution set of polynomial systems," in Proc. 39th IEEE Conf. Decision Control, Sydney, Australia, 2000, pp. 1501-1506.

[9] G. Chesi, A. Garulli, A. Tesi, and A. Vicino, "Characterizing the solution set of polynomial systems in terms of homogeneous forms: An LMI approach," Int. J. Robust Nonlinear Control, vol. 13, no. 13, pp. 1239-1257, 2003.

[10] D. Henrion and J. Lasserre, "Detecting global optimality and extracting solutions in gloptipoly," in Positive Polynomials in Control, ser. Lecture Notes in Control and Information Sciences, D. Henrion and A. Garulli, Eds. London, U.K.: Springer-Verlag, 2005, no. 312.

[11] G. Chesi, "Domain of attraction: Estimates for non-polynomial systems via LMIs," in Proc. 16th IFAC World Congr. Autom. Control, Prague, Czech Republic, 2005.

[12] G. Chesi and Y. S. Hung, "Trajectories bounds for nonlinear systems: A BMI approach," in Proc. 46th IEEE Conf. Decision Control, New Orleans, LA, 2007, pp. 4001-4006. 